\title{
Antoine Culioli - « Toute théorie doit être modeste et inquiète "
}

Entretien avec Almuth Grésillon et Jean-Louis Lebrave

\section{Almuth Grésillon et Jean-Louis Lebrave}

\section{(2) OpenEdition}

\section{Journals}

\section{Édition électronique}

URL : http://journals.openedition.org/genesis/1071

DOI : 10.4000/genesis.1071

ISSN : 2268-1590

\section{Éditeur :}

Presses universitaires de Paris Sorbonne (PUPS), Société internationale de génétique artistique littéraire et scientifique (SIGALES)

\section{Édition imprimée}

Date de publication : 15 novembre 2012

Pagination : 147-155

ISBN : 978-2-84050-869-4

ISSN : 1167-5101

\section{Référence électronique}

Almuth Grésillon et Jean-Louis Lebrave, « Antoine Culioli - « Toute théorie doit être modeste et inquiète » », Genesis [En ligne], 35 | 2012, mis en ligne le 15 novembre 2014, consulté le 21 juin 2020. URL : http://journals.openedition.org/genesis/1071 ; DOI : https://doi.org/10.4000/genesis.1071 
Antoine Culioli " Toute théorie doit être modeste et inquiète "

\author{
Entretien avec Jean-Louis Lebrave et Almuth Grésillon
}

Antoine Culioli est une figure majeure de la linguistique contemporaine. Depuis plus de quarante ans, il développe une théorie connue sous le nom de "Théorie des opérations énonciatives». Son objet est l'étude de l'activité de langage appréhendée à travers la diversité des langues, des textes et des situations. Ses travaux ouvrent des perspectives sur d'autres champs de recherche et intéressent plus généralement l'ensemble des sciences humaines. C'est dans cet esprit qu'il a bien voulu nous accorder un entretien, qui s'est déroulé le 20 juin 2011. L'échange a porté sur les voies qui l'ont conduit à la linguistique, sur sa manière de travailler et de progresser dans l'élaboration de sa théorie, mais aussi sur la compatibilité éventuelle de celle-ci avec la critique génétique.

Jean-Louis Lebrave et Almuth Grésillon - Merci, cher Antoine Culioli, de nous accorder cet entretien. À notre première prise de contact, vous avez dit très clairement que vous ne souhaitiez pas répondre à des questions préparées à l'avance et que vous ne vouliez pas "que l'on retouche le texte ", qui doit rester tel que vous l'aurez produit. Pourquoi cette insistance, cet attachement à la lettre dite?

Antoine Culioli - On m'a souvent dit qu'à travers les transcriptions fidèles on entendait ma voix, ma prosodie. Le texte, c'est finalement une texture, c'est quelque chose de beaucoup plus compliqué que du linéaire. Dans la Lettre à Hérodote, Épicure parle de $\sigma \nu \mu \pi \lambda o \kappa \eta ́$ à propos des atomes, ce qui a été traduit en anglais par interlace, « entrelacer ». C'est vraiment ce genre de choses. De même que Marcel Mauss, le spécialiste du don, de l'échange, parle du fait social comme d'un " échange total », je dirais que les faits de langage, l'activité de langage, c'est une activité totale, qui est à la fois un travail d'interlocution et un travail d'intersubjectivité. Voyez l'exemple très simple, et totalement ignoré, de la formation du conditionnel, qui est une forme du fictif par excellence.

Vous avez d'un côté l'infinitif, venir, ensuite il y a le verbe avoir, puisque ça vient de habebat, et puis vous avez l'imparfait ou le prétérit. On part d'une base, l'infinitif, qui n'est pas implanté, pas situé. Ensuite, le verbe avoir introduit un hiatus. Puis il y a une forme qui permet l'insertion, et c'est là qu'on a la deuxième personne (fig. 1).

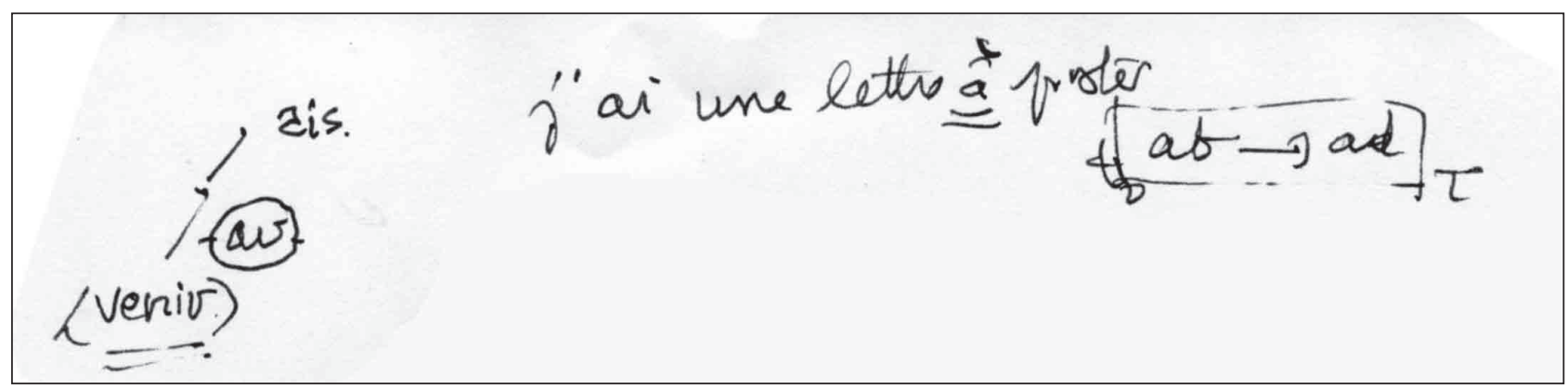

Fig. 1 : Dessin réalisé par Antoine Culioli au cours de l'entretien 
Il y a des gens qui ne comprennent pas pourquoi je fais tant d'étymologies. Ce n'est pas parce que je veux imiter tel ou tel philosophe, mais tout simplement parce que le travail philologique est un travail qui vous fait remonter vers des vestiges. Lorsqu'on trouve un bout d'os dans un désert, on ne s'étonne pas qu'on nous explique tout ce qu'il permet de montrer : la philologie, c'est ça. Par exemple, pour des germanistes, je n'ai jamais vu personne s'étonner du fait qu'en allemand, schier signifie d'un côté pur, absolu, et de l'autre, presque. Pour en rendre compte, vous ne pouvez pas avoir une théorie scalaire où vous avez une échelle, il faut une théorie vectorielle, c'est-à-dire que ça projette. Et là-dessus il suffit de lire ce que Jankélévitch a écrit sur les travaux de Georg Simmel ${ }^{1}$ et de voir qu'il y a toujours un mouvement au-delà, et quand il n'y a pas de mouvement au-delà, c'est qu'il y a le [ne...] plus, c'est fini. Vous savez comment on dit exister en suédois ou en norvégien? Vous prenez le verbe être, en suédois c'est vara (le Wesen de l'allemand bien sûr), et puis vous ajoutez $\mathrm{til}$, qui veut dire dans un cas comme ça « quelque chose de plus », ça vous projette en avant, c'est ce que vous trouvez aussi dans le mot Ziel en allemand. Tout ça, il faut le sortir, sinon on n'y pense pas. Nous sommes totalement non conscients de notre activité mentale, et en un sens c'est une bonne chose. Si les hommes, homo sapiens, et même sapiens sapiens, s'étaient brusquement dit « je vais faire de la linguistique », ils étaient fichus. Ils n'auraient jamais plus parlé.

J.-L. L. et A. G. - Vous avez évoqué la philologie et la linguistique. Vous êtes entré à l'École normale supérieure en 1944, et vous avez choisi de préparer l'agrégation d'anglais. Pouvez-vous nous rappeler dans quel contexte vous avez fait vos études et comment ensuite le jeune agrégé d' anglais est devenu linguiste?

A. C. - J'ai passé le certificat de philologie anglaise en écoutant les réponses des autres à l'oral - vraiment, le niveau était lamentable. Après l'agrégation, j'ai été nommé assistant de philologie anglaise. Les gens ont critiqué ma pédagogie folle. En fait, elle a sûrement été utile, parce que je rencontre encore maintenant des gens qui me disent que c'est une des rares fois où ils ont eu l'impression de voir un professeur qui pensait. Je réfléchissais au fur et à mesure, en me présentant si j'ose dire dans ma faiblesse, avec modestie et inquiétude. J'ai suivi les cours de Mossé au Collège de France. Il y avait aussi Fourquet, et Jolivet pour l'islandais. Avec Mossé, j'ai tout fait, le gotique, le frison, etc., toutes les langues germaniques. Parallèlement, j'ai fait ma thèse avec Émile Pons, un spécialiste de Swift, sur la disparition du subjonctif en anglais médiéval. La thèse secondaire était sur Dryden traducteur de Chaucer et de Boccace.

J.-L. L. et A. G. - Donc, c'est vraiment la philologie qui vous a conduit vers la linguistique! Quant à nous, lorsque, jeunes germanistes linguistes, nous avons commencé dans les années soixante-dix et quatre-vingt à étudier les manuscrits littéraires, nous avons dû rompre avec une certaine philologie, la philologie éditoriale allemande qui depuis le XIXe siècle avait élaboré le modèle des éditions historico-critiques. Or, nous ne voulions pas établir des appareils de variantes qui n'expliquent rien sur la dynamique de l'écriture, mais comprendre le processus lui-même.

A. C. - Oui, c'est comme si vous faisiez un genou sans tendons, sans liquide de synovie. Alors, bien sûr, vous pouvez toujours le mettre avec des ficelles ou avec des fils de fer, mais c'est un peu comme les gens qui n'aiment les papillons qu'épinglés, et ces gens risquent de vous dire que vous êtes dans le fantasme. Mais comme dit Husserl pour la géométrie, il faut avoir une freie Phantasie dès que vous introduisez de la vie, du mouvant.

J.-L. L. et A. G. - Justement. Nous avons souvent eu l'intuition qu'entre votre théorie des opérations énonciatives et notre pratique de linguistes généticiens sur les manuscrits, il y avait une affinité induite par le "langage en action ", le bougé, le jamais fini, ces variations qui supposent un invariant...

1. Georg Simmel, La Tragédie de la culture et autres essais, Introduction de Vladimir Jankélévitch, Marseille/Paris, Rivages, 1988. 
A. C. - Ah oui, oui, je le pense. Ou bien on dit qu'on n'y a pas accès, ou bien on dit que nous créons à un moment donné une zone où nous voyons qu'il se passe des choses, où nous pouvons construire le problème qui permet éventuellement de rendre compte de ce que nous trouvons : à ce moment-là, nous avons une position qui nous permet de progresser.

J.-L. L. et A. G. - Pouvez-vous nous en dire plus ? Car face à cette liberté de complexifier, de construire, nous, nous avons la contrainte, précisément, du corpus contraint.

A. C. - Bien sûr. Je vais vous donner un exemple qui me vient brusquement à l'esprit. À un moment donné j'ai étudié les emplois de $a ̀$ et de de en français dans par exemple « je me force à ne rien dire », « je suis forcé de ne rien dire », « je me suis forcé à ne rien dire », etc. Je leur attribue ce que j'appelle une forme schématique, et schématique, ça vient tout simplement de skema, qui était le mot d'Aristote pour ce que les atomistes appelaient rythmos, le rythme. Sur cet exemple de forcer, quelqu'un dans le séminaire m'a dit : "Oui, mais il y a "Objets inanimés avez-vous donc une âme / Qui s'attache à notre âme et nous force d'aimer ?" et non pas "nous force à aimer". » Moi, si on me donne un exemple, je dois en rendre compte. Alors j'ai repris Lamartine. « Nous forcer à aimer », ça veut dire que l'âme ferait pression, parce qu'en français, $a ̀$ c'est parce qu'il y a pression, ça vient de $a b, a d$; quand vous avez $d e$, c'est autre chose, dans un cas comme ça, $d e$, ça veut dire que c'est un fait. Et donc vous vous apercevez que forcé d'aimer, ça n'est pas du tout un contre-exemple, ça veut dire une pression spirituelle et non pas quelque chose qui serait une contrainte de l'ordre de la force, même s'il y a forcé...

J.-L. L. et A. G. - Un autre trait nous frappe beaucoup dans votre théorie. Vous parlez de possibilités de ratés, d'ambivalences, de flou, ce n'est pas très loin de nos ratures, de nos accidents, du processus scriptural avec ses ajouts, substitutions, suppressions, c' est-à-dire tout ce qui relève du mouvement, de la dynamique...

A. C. - Oui, bien sûr, je ne sais pas si ça vous permet d'arriver à quelque chose, mais cela donne une idée du travail qui a été effectué. Je me souviens de manuscrits poétiques de Valéry, son premier jet était affreux. Et puis au fur et à mesure, il changeait, il enlevait quelque chose, il mettait autre chose, et il y avait un moment où ça se stabilisait, où vous voyiez que ça devenait quelque chose. Pourquoi n'auriez-vous pas le droit de dire qu'à un moment donné, une poésie est bonne ou est mauvaise ? Il y a des poésies qui vous émeuvent et d'autres qui au contraire vous laissent entièrement froid ou bien vous font ricaner. Vous avez bien le droit de le dire tout de même.

J.-L. L. et A. G. - En fait, nous nous interdisons de dire que c'est manvais parce que nous avons dû batailler très activement contre ce qu'on pourrait appeler la tentation téléologique de la critique traditionnelle, qui postule que tout processus de création évolue vers une œuvre parfaite. Les manuscrits d'écrivains montrent surabondamment que ce n'est pas le cas : la création procède par ratés, par essais qui avortent, par recommencements ; c'est donc pour nous un principe heuristique de ne pas partir de l'idée que le poème aurait commencé par être assez médiocre, et qu'il serait progressivement devenu bon. Et inversement, ce que vous dites fait penser à la boutade de Heine : on lui avait demandé d'où lui venait son génie poétique, et il avait répondu : "c'est simple, j'ai travaillé », « ich habe gearbeitet».

A. C. - J'ai publié un article, "Sur quelques contradictions en linguistique ${ }^{2} »$; vous pourriez ainsi parler de «quelques contradictions en génétique », au sens où justement, il y a à la fois l'inspiration et le travail artisanal - mais pas à la manière de Boileau : «Vingt fois sur le métier remettez l'ouvrage, polissez-le sans cesse et le repolissez ", ça c'est autre chose, ça sentait l'huile de coude, toute cette poésie-là...

J.-L. L. et A. G. - On dit que Virgile composait une vingtaine de vers dans le calme de la nuit, qu'au matin il appelait son secrétaire auquel il dictait ces vingt lignes, et qu'il passait le reste de la journée à les réécrire, à les

2. «Sur quelques contradictions en linguistique », dans Communications, vol. XX, 1973, p. 83-91. 
polir - c'est l'origine des vers de Boileau. On a donc bien le mélange de l'inspiration et du travail.

A. C. - Virgile est tout de même supérieur à Boileau ! Le précepte «polissez-le » veut dire qu'on a pris conscience du travail, mais même dans le cas inverse, le travail existe, même si on n'en a pas conscience. Le problème c'est que, évidemment, l'activité mentale vous échappe. Oui, elle échappe, elle échappera toujours. Toute l'imagerie médicale, toutes les cartographies du cerveau ne permettront jamais de voir autre chose qu'un central téléphonique. Si on voyait tout, qu'est-ce qu'on verrait ? On verrait les fils, mais on ne verrait pas ce qui passe dans les fils.

J.-L. L. et A. G. - Ceci nous amène à vous poser la question suivante. Dans un chapitre qu'elle a écrit pour un volume coordonné par Michel Fayol sur Production du langage ${ }^{3}$, Stéphane Robert suggère que s'il y a une théorie linguistique susceptible de contribuer à une meilleure connaissance de la production verbale, c'est chez vous qu'il faut la chercher. Nous le pensons aussi parce que votre théorie est à notre connaissance la seule qui parte du dynamique.

A. C. - Dans une conférence que j'ai faite récemment d'ailleurs, elle a été enregistrée et sera publiée à l'Inalco -, je faisais la comparaison avec la théorie des jeux de Morgenstern et von Neumann qui disaient « ce que nous faisons est statique, et il faut passer à la dynamique ${ }^{\star} »$. Or de plus en plus, j'étais passé par des choses de ce genre. Par exemple, il y avait la topologie, qui est par excellence l'art de la déformation. Mais on ne voit pas pourquoi on ferait du langage une espèce de dispositif à faire des pâtes fraîches... Après il y avait le transformationnel, c'est Harris qui l'a inventé. Mais moi, je regarde quelles sont les opérations qui permettent de passer d'une forme à une autre, ce qui n'est pas du tout la même chose que la paraphrase, même si c'est voisin en apparence. Quand je prenais un exemple, je disais « Jean a un trou à sa poche », «Jean, sa poche, elle a un trou », mais on ne peut pas dire «Jean un trou est à la poche », des choses comme ça. Autre exemple, la manière de renforcer une affirmation positive : à la question "Est-ce que tu es content?", on peut répondre par exemple « Je veux » ou «Plutôt ». Ce «plutôt » suppose du dynamique, il envoie vers un attracteur ; sinon ça va vous envoyer au contraire vers zéro, c'est-à-dire dans l'á $\pi \varepsilon i \rho \omega v$, c'est ce qui est hors mesure, hors du mesurable, hors du dicible, etc. C'est de la langue ordinaire, pas «the cat is on the mat».

J.-L. L. et A. G. - Mais nous, nous n'avons pas affaire à de l'oral, nous devons nous débrouiller avec l'écrit seul, sans trace de l'intonation, de la prosodie, etc., ce qui est une réelle frustration.

A. C. - Il faut que vous le fassiez tourner dans votre tête, et souvent, vous allez voir apparaître des choses auxquelles vous ne pensiez pas. Par exemple, l'histoire de je veux, c'est facile. «Je le veux », « je veux quelque chose », « je lui en veux », « je m'en veux à moi-même ». Vous coupez le. Il n'y a plus de chose sur laquelle porte votre volonté. « Je m'en veux ». Vous enlevez me. Vous passez à « il en veut » - attention, il faut faire la théorie de en, et on voit que ça veut dire «il en veut, avec ses tripes », c'est vraiment son intérieur, toute sa tension musculaire qui est là. Vous coupez tout ça, il vous reste « Je veux », et il vous reste la prosodie, «Je veux ». Et c'est pour ça que d'ailleurs très souvent, avec une sorte de rhyming slang, les gens ajoutent « mon neveu », et après ils disent « Tu parles Charles ».

J.-L. L. et A. G. - Il y a une idée toute faite sur la proximité de votre théorie avec une théorie de la production écrite: puisque nous travaillons sur la production écrite, il irait de soi d'utiliser directement votre théorie des opérations énonciatives. Or, ce que vous nous avez dit depuis le début de cet entretien ne paraît pas forcément relever de l'énonciation au sens où on la manipule d' habitude.

A. C. - Oui, l'énonciation, je l'ai prise au sens strict. Je l'ai tirée en fait de toute la théorie stoïcienne, mais mélangée

3. Stéphane Robert, « Modèles linguistiques de production », dans M. Fayol (dir.), Production du langage, Paris, Hermès, 2002, p. 66-86.

4. Voir aussi Antoine Culioli, Pour une théorie de l'énonciation, Paris, Ophrys, 1990-1999, t. II, p. 12. 
d'atomisme aussi pour certaines choses. C'est vraiment chez les Grecs que je l'ai trouvée. Je prends la lettre 117 des Lettres à Lucilius, où Sénèque parle d'abord de l'ordre du corps : «Corpus est, quod video, cui et oculos intendi et animum. Dico deinde : Cato ambulat. Non corpus, "inquit", est, quod nunc loquor, sed enuntiativum quiddam de corpore, quod alii effatum vocant, alii enuntiatum, alii dictum ${ }^{5} »$; c'est l'essence des choses, le corpus, on peut dire aussi que c'est du domaine sensori-moteur. C'est donc un existant sensori-moteur, et non pas un existant fictif. De l'autre côté, il y a un enunciatum, un énoncé, un dictum. Pour moi, il y a trois niveaux. Dans le premier c'est votre corps ; dans le second c'est à la fois votre corps et la représentation que vous vous faites à partir de quelque chose que vous percevez par vos sens ; le troisième est proprement mental, puisque c'est entre une chose et une autre, avec une béance.

J.-L. L. et A. G. - Nous nous sommes demandé aussi s'il était possible de considérer certaines réécritures comme relevant de ce que vous appelez l'épilinguistique...

A. C. - Mais bien sûr. Il y a un travail qui se fait qui échappe au locuteur et qui fait qu'à un moment donné il se dit «c'est pas ça ». Après, quoi qu'on dise, il y a un moment où on considère que «ça y est ». Moi, je n'ai pas de conseils à donner, mais vous pourriez être beaucoup plus, ne disons pas polémiques, mais hardis sur le plan de la théorisation, en expliquant pourquoi vous faites ce que vous faites. Parce que, il n'y a pas à dire, sinon, c'est inaccessible pour toujours, et dans ce cas, eh bien, ce n'est pas la peine. Vraiment, le dilemme est très clair, là. Vous pensez, vous espérez, du moins vous souhaitez, ou vous suggérez que vous pouvez accéder, à quoi ? au Graal ?

J.-L. L. et A. G. - Non..., mais aller un petit peu plus loin dans la reconstruction du processus écrit, ça oui. D'ailleurs, pour désigner ce qui se passe lors des réécritures, nous employons le terme d' "opération", notion fondamentale dans votre théorie, mais qui ne signifie peut-être pas la même chose, car nous n'avons pas vraiment accès aux opérations que vous construisez.

A. C. - Vous pouvez employer le mot opération, si vous voulez, en disant que « par abus de langage, j'appellerai ça opération », mais effectivement, vous n'avez pas accès aux opérations elles-mêmes, puisque vous êtes en train d'essayer de refaire des trajets qui ont non seulement disparu depuis longtemps, mais qui vous échappent, et qui nous échappent, et qui nous échapperont. Alors il faudrait peut-être dire, après tout, un travail, comme on dit «le travail à l'œuvre »; je ne sais pas s'il est simplement mental, parce qu'il est à la fois affectif...

J.-L. L. et A. G. - Il est aussi concret, nous disons souvent "opération » pour désigner une addition ou une suppression, un remplacement, ou un déplacement, qui correspondent à la traduction matérielle d'une opération immatérielle.

A. C. - Je vous propose ceci : vous mettez opération, vous dites «par abus de langage », et vous expliquez l'abus de langage, parce qu'il s'agit à la fois, effectivement, d'une opération - quand on vous opère, heureusement que c'est matériel -, et puis d'un autre côté, il y a par exemple les opérations arithmétiques : «3+5-4, ça fait combien?», ce ne sont pas des opérations matérielles.

J.-L. L. et A. G. - Si nous insistons sur ce point, c'est parce que, dans la pratique, un certain nombre de gens ne prennent pas de précautions, et passent spontanément de l'opération graphique qui consiste à barrer un mot à "c'est une opération mentale, et j’y ai accès - la voilà »!

A. C. - C'est de la naïveté. Pour moi, vous avez affaire à des gens qui ne comprennent pas l'abstraction, ils ne voient même pas dans certains cas pourquoi vous vous posez ces problèmes, pourquoi vous vous compliquez la vie.

5. «C'est un corps que je vois, qui occupe mes yeux, ma pensée. Ensuite je dis : "Caton se promène." Ce que je dis là n'exprime pas un corps ; mais est expression déclarative d'un état corporel appelée par les uns un "prononcé", par les autres un "énoncé", par d'autres un parlé ( $\lambda \varepsilon \kappa \tau o ́ v)$ », Sénèque, Lettres à Lucilius, t. V, texte établi par François Préchac et traduit par Henri Noblot, Paris, Société d'édition « Les Belles lettres », 1964, p. 49. 
J.-L. L. et A. G. - Ils ne comprennent même pas ce que nous voulons dire lorsque nous leur expliquons que notre objet n'est pas déjà là, qu'il faut le construire...

A. C. - J'ai commencé, vous vous en souvenez peut-être, dans l'article de 1968 des Cahiers pour l'analyse ${ }^{6}$, par dire « voilà ce que je fais, voilà ce que je vais faire »; il y avait pratiquement tout dedans. Les gens m'en ont voulu, j'ai eu des réactions absolument invraisemblables ; déjà quand je mettais "activité de langage ", ils m'ont critiqué. Puis, un collègue germaniste m'a dit un jour : «Vous inquiétez les étudiants. » Il m'a dit ça comme ça, gentiment, sans se rendre compte de l'énormité. On m'a dit aussi: «Vous prenez des risques. »

J.-L. L. et A. G. - Mais l'inquiétude, c'était presque un compliment. Inquiéter les esprits, c'est les empêcher de dormir.

A. C. - L'inquiétude philosophique. C'est ce que Locke appelait la philosophical uneasiness. Comme je le dis depuis longtemps : " Toute théorie doit être modeste et inquiète ${ }^{7}$. » Là, ça sera sans doute un de vos problèmes, et ça l'est peut-être déjà, mais en tout cas, moi, ça m'a accompagné toute ma vie. Mais alors, je m'en moque. Mais alors complètement. C'était à l'époque bénie où l'EHESS a été créée à partir de la VIe section. On se rencontrait entre mathématiciens, anthropologues, etc. Moi-même, j'ai travaillé avec des linguistes comme Rygaloff, spécialiste de chinois, avec le psychologue François Bresson, mais aussi avec Laplanche et Bourguignon. On a travaillé sur l'aphasie, sur les schizophrènes. À chaque fois, quelle satisfaction ! J'arrivais comme ça, avec ma petite théorie et puis pfuitt, c'était balayé en un rien de temps. Quand vous avez une pauvre femme schizophrène qui dit «Je suis une femme comme une autre », puis elle dit «Je suis une femme comme les autres », c'est extraordinaire. "Une femme comme une autre », c'est banal, et «Une femme comme les autres », c'est «j'ai droit à être une femme comme une autre », et tout ça provient du pluriel et c'est ça qui vous donne à ce moment-là la différence entre d'un côté « le droit à », et d'un autre côté la banalité.
J.-L. L. et A. G. - Oui, mais de nouveau, ce sont les surprises extraordinaires de l'oral...

A. C. - Oui, vos auteurs, évidemment, ils sont morts, les pauvres. Mais quand même, il faut avoir cette espèce de malléabilité, de plasticité et de curiosité.

J.-L. L. et A. G. - Vous avez parlé de ce que nous aimerions appeler votre curiosité native, mais en même temps, pour nous qui sommes d'une génération postérieure à la vôtre, nous avons le sentiment que vous êtes devenu linguiste à une époque exceptionnelle où tout était à découvrir et à inventer.

A. C. - Ah oui, absolument, c'est ce que m'a dit Milner : « Vous étiez devant un terrain vierge. »

J.-L. L. et A. G. - Oui, un terrain vierge. Vous construisiez votre théorie, mais en même temps, vous étiez à l'écoute de tout et de tous. Si bien que, par exemple, dans les années quatre-vingt, vous avez accepté de préfacer deux ouvrages que nous avons publiés, La Genèse du texte. Les modèles linguistiques (Éditions du CNRS, 1982) et La Langue au ras du texte (Presses universitaires de Lille, 1984), geste qui témoigne à la fois de votre ouverture d'esprit et de votre générosité intellectuelle. Récemment, nous avons retrouvé, non sans émotion, le manuscrit (pas l'original, mais une photocopie!) de l'une de ces préfaces, mis au net dans une belle écriture soignée, presque calligraphique (fig. 2). Si nous vous le montrons aujourd' hui, plus de trente ans après, c' est pour vous poser une question : que fait Antoine Culioli de ses manuscrits? Les a-t-il gardés, archivés?

A. C. - Tiens, je ne connaissais pas ça ; belle écriture, hein ?

J.-L. L. et A. G. - C'est bien pour cette raison que nous vous le montrons, et que nous vous interrogeons sur vos manuscrits...

6. Il s'agit de l'article intitulé « La formalisation en linguistique », dans Cahiers pour l'analyse, ${ }^{\circ}$ 9, 1968.

7. Voir Antoine Culioli, Pour une linguistique de l'énonciation, Paris, Ophrys, 1990-1999, t. III, p. 174. 
énonciatif et qui ne ramène pas l'activité rignifiante à l'empaquetage codé d'une intention claire et arrêtée, une linguistique des ajustements énonciatifs et non "une lingristiveue de la communication ma. nifeste et réussie, oir l'on ne puogramme que des puedications heu. reuses", si l'on me pardonne cette auto-citation.

Je me permettrai de pulonger ces remarques personnelles, en $x^{\prime}$ gralant que j'ai moi-même fait l'expizience de l'inefficacité d'une certaine linguistyue, alno que je travaillais sur le langage des poy. chotiques avec jean Laylanche, et de fa con quo générale, dès que low s'occupe des textes tels $q \mu^{\prime}$ i is sont et non pas tels que, aux yeux du linguiste, ils dev raient être. D'où l'entre pise d'elaboration d'une Théorie fromelle des opérations juédicatives er énanciatives. Or, les manucrits, comme tont ensemble complexe d'états successifs en voie de stablilisation, forcent à la même remise en question radicale d'une certaine technique linguistique, erceci à un tiple titre. D'un côté, les textes forment un corpus contraint qui neot ni un é. chantillon (ou it est échantillon représentatif de hi-même) ni un ensemble extensible et manipulable, cor it ne o'agit pas ici de simulation. Ainsi, on ne peut pas travailler directement sur les variations, analypées dans lew discontinuité, comme on peut travall. ler sour des classes d'énoncés équivalents, où lion peut contióler les modulations et leo déformations référentielles. En second lieu, l'écriture, comme la lecture, ne pent se ramener à un enchaînement strictement linéaire d'opérations oucceorives qui produiraient du texte et des significations arsociées. In d'autres termes, le manuscit porte latrace d'opérations qui ne sont ni ordonnées ni homogènes, et nons ne pousons pas eopérer remonter le cours des opérations, pas une 4. médiation nozmative, comme, parfoiro, devant un écheveau emmêlé, on erpère tirer sour un fil pow que tont vienne. Pourquis, dano ces con ditions (erce sera une trosième remaque) attii buer à la linguishique un quelconque statut dans cette recherche oü doivent concousir tontes leo discilines qui, d'une faços ou d'ine autre, traitent du symbolique? y-a.r.il quelque sens à croire que la linquistique pourra, ne serait-ce que le temps d'un recueil, faire, à elle seule, cequ'un faisceau de recherches réusint à grand' vire à entrevoir? En un mot, if faut bien de l'optimisme pour eopérer eomul relier représentants (traces textuelles) et représentrations symboliques, à partír de ce bougé que sont les variantes, avec le seul recours à la linguishique. Or, l'ow rage montre qu'en de pit des obstacles, une démarche ar. gumentée se fonde, une discipline complexe s'affermit, et l'on n'a, en refermant le liore, ni une impremion de banalite ni le sentiment qu'on aurait dejlogé un grand appareil pour un résultat mince. Iest évident que, en disant cela, je nuis sans donte juge et partie. on ne m'aurait pas de mandé d'écrire une pueface, si lion ne sonp sonnait chez moi une cerbaine sympathie. mais cet interêt m'acrée davantage diobligations dans malec. ture et une difiance à l'égard de mes propes jugements. Ces piecautions prises, il reote que le recueil tient tien, d'autant que (eresst un mérite oupplémentaire) it eot un peu comme un ma. nuel par la nature exe mylaire des thimes trait's cinsoi que par le soxci d'esyliciter les aspects théniques et techniques de l'entep pise. Enoutre, it n'y a pas de redites, même si l'on trouve quelques inté.

Fig. 2 : L'écriture manuscrite d'Antoine Culioli dans sa préface au volume La Genèse du texte. Les modèles linguistique (1982)

A. C. - Je jette - mais j'ai aussi des dossiers qui restent : ce sont d'une part des papiers, des documents sur les langues, que j'ai rassemblés et dont je ne suis pas l'auteur, et d'autre part mon propre travail. Oui, je garde, j'augmente au fur et à mesure. À un moment donné, je fais des opérations, pour voir si ça marche. Je fais des gestes, des croquis. Quand vous esquissez un tore, vous allez mettre un carré avec des flèches qui vont dans un sens, d'autres dans un autre sens, des choses comme ça. C'est très difficile à rendre. Alors je fais des papiers, je les recommence, je les re-recommence, et maintenant, quand je pars en Corse, je fais attention : je pars avec treize kilos, je reviens avec quinze. Denis Paillard m'a dit : « Surtout ne jetez rien, absolument. »

\section{J.-L. L. et A. G. - Nous sommes bien d'accord!}

A. C. - Mais c'est mal fichu parce que parfois une pile, ça s'effondre, alors je remets les papiers en place comme je peux.

J.-L. L. et A. G. - Mais de tel ou tel article, il doit bien exister les esquisses, les brouillons, les versions successives, non? Ce serait une mine pour nous!

A. C. - Dans certains cas c'est évident. Par exemple, j'ai travaillé sur puisque. Puisque est extrêmement intéressant dans la mesure où il n'existe pas dans toutes les langues. En allemand, il n'existe pas. À la rigueur $d a$, peut-être, 
mais la différence avec weil est moins nette que celle entre puisque et parce que. Et en suédois, ça existe, mais sans trop exister ; j'ai fait toute une étude là-dessus. Et puis, au fur et à mesure j'en découvre d'autres, dans d'autres langues. Par exemple, il est intéressant de voir que, en islandais, il n'y avait pas de puisque. L'islandais en a fabriqué un assez tôt, en employant le mot fyrst, avec un $y$, qui veut dire premièrement. Et en fait, ce n'est pas premièrement. Ça veut dire : « ceci étant posé, et étant stable, il s'en suit que ». Puis vous avez since en anglais, en italien c'est gia che, c'est gia, c'est-à-dire iam du latin, déjà ; si vous dites déjà, ça veut dire qu'il y a un désormais. Si déjà est acquis, ça veut dire que désormais l'acquis fait partie de ce qui se déroule et qui va se transformer, etc. Oui, vous voyez, il y a un gros dossier sur puisque. Mais pour revenir à fyrst, je ne savais pas comment l'utiliser. Alors, j'ai téléphoné à l'ambassade d'Islande, pour le leur demander. Et après j'ai lu Mireille de Mistral, et je me suis aperçu qu'en provençal, pour dire puisque, on dit d'abord. «D'abord je vous ai vu », ça veut dire « dès que je vous ai vu, dès lors que je vous ai vu, du moment que je vous ai vu, dans la mesure où je vous ai vu, puisque je vous ai vu ». Et voyez, une fois de plus, vous vous apercevez que cette opération dont je viens de vous parler, je ne dirai sûrement jamais qu'elle est universelle, ça alors je laisse ça à d'autres...

\section{J.-L. L. et A. G. -Oui, mais vous dites tout de même qu' il} $y$ a des invariants.

A. C. - Je dis que dans les textes, dans ce que j'ai vu, puisque, ça peut ne pas exister, comme justement en allemand, où vous ne pouvez pas l'avoir, c'est tout. Et dans certains cas vous voyez combien la langue tâtonne.

J.-L. L. et A. G. - Et encore une fois, vous ne vous êtes jamais posé la question: "Qu'est-ce que je ferai de tous ces manuscrits?»

A. C. - Eh bien, je vais essayer de faire un tome IV, et puis, si Dieu me prête vie, je voudrais faire un tome V, avec des choses comme ça. Mais le problème, c'est la présentation. Je le ferais en dessin animé, ce serait beaucoup plus drôle qu'au tableau !
J.-L. L. et A. G. - Nous vous avons dit tout au début que cet entretien devait paraître dans un numéro de notre revue, Genesis, qui sera coordonné par Irène Fenoglio. Elle travaille sur les manuscrits de Benveniste qui sont à la BnF. Et justement, concernant Benveniste et son rôle dans l'histoire des concepts linguistiques, vous avez écrit : "Il nous faudrait étudier les notes de travail de Benveniste, connaître sa bibliothèque, ses lectures, comparer ses opinions à celles de Jakobson et de Kurilowicz. Il est clair qu'un tel travail supposait une compétence qui me fait défaut, mais aussi un accès aux documents qui n'était pas possible jusqu'à maintenant ${ }^{8}$. » Donc c'est vous qui, très tôt, aviez déjà mis le doigt sur l'intérêt des manuscrits pour les recherches futures. Ajoutons, pas seulement les manuscrits de Benveniste, mais aussi les vôtres...

A. C. - Il y a beaucoup de mes textes, notamment de mon séminaire, qui depuis un certain temps ont été enregistrés.

J.-L. L. et A. G. - Il y a eu aussi un film avec vous, sur vous.

A. C. - Oui, c'est Rodriguez qui avait fait ça en 1994. Il est même passé à la télévision, à un moment qui me permettait d'avoir la gloire éternelle, à 5 h 30 du matin ! Et ce Rodriguez, qui a fait un certain nombre de films sur toute une série de gens, a enregistré des kilomètres de bobines pour faire un second film sur moi, mais il a tellement de rushes, je ne sais pas s'il arrivera à les caser en cinquante-deux minutes. Mais encore une fois, oui, il y a des séminaires ou des conférences qui ont été enregistrés ou même filmés.

J.-L. L. et A. G. - Qu'avez-vous pensé lorsque Claudine Normand a suggéré qu'en fait, votre théorie était une poétique ${ }^{9}$ ?

8. Voir A. Culioli, « Théorie du langage et théorie des langues », dans Actes du colloque de Tours, 1983, diffusé par les éditions Peeters, Louvain, p. 77-83.

9. Voir Claudine Normand, « La théorie d'Antoine Culioli, une poétique », dans Dominique Ducard et Claudine Normand (dir.), Antoine Culioli. Un homme dans le langage (colloque de Cerisy), Paris, Ophrys, 2006, p. 361-366. 
A. C. - Jean-Claude Chevalier, lui, a dit que j'étais un conteur. Quand j'étais encore en Corse, j'avais une grand-mère qui me racontait des histoires de Corse, des histoires, ce qu'on appelait fola - qui vient de fabula d'ailleurs - et j'avais une mère qui venait d'une famille qui faisait spontanément de belles métaphores - et il y avait un poète dans le village...
J.-L. L. et A. G. - Bref, vous êtes un conteur philologique. La conjugaison des deux, une théorie proche d'une poétique, incarnée par un conteur philologique, voilà qui donnerait une image assez juste de vous. Les affinités qui nous lient passent sans doute aussi par là. Après tout, nous nous occupons de poètes.

A. C. - Oui, c'est le gai savoir, sous une autre forme.

Almuth GréSILlon est directrice de recherche émérite à l'ITEM (CNRS-ENS) et responsable de la revue Genesis. Elle est l'auteur d'un ouvrage de référence, Éléments de critique génétique. Lire les manuscrits modernes (Paris, PUF, 1994), ainsi que de La Mise en œuvre. Itinéraires génétiques (Paris, CNRS Éditions, 2008) et de nombreuses autres études de génétique littéraire.

almuth.gresillon@ens.fr

JEAN-LOUIS LEBRAVE est directeur de recherche émérite à l'ITEM (CNRS-ENS). Il a travaillé notamment sur les manuscrits de Heine, de Proust, de Flaubert, de Barthes, de Vitez. Outre des publications sur la théorie génétique, il s'est intéressé à l'hypertexte (« Hypertextes - Mémoires - Écriture », Genesis, n 5 , 1994), aux traitements informatiques des données manuscrites et à l'incidence des technologies de l'information sur l'écriture (Texte et ordinateur : les mutations du lire-écrire, Espace européen, 1991, avec J. Anis ; «Retour vers le futur. Supports anciens et modernes de la connaissance », Diogène, n 196, 2001, avec J.-G. Ganascia). Par ailleurs, il s'est attaché à l'analyse des dimensions psycholinguistiques de la critique génétique.

jean-louis@lebrave.name 